\title{
Impact of additional intravenous methylprednisolone pulse therapy on the quality of life in patients with dysthyroid optic neuropathy
}

\section{Maryla Pelewicz}

Student Scientific Group "Endocrinus", Department of Internal Medicine and Endocrinology, Medical University of Warsaw, Poland<smiles>[C+]1[CH+]CC1</smiles>

\section{Joanna Rymuza}

Department of Internal Medicine and Endocrinology, Medical University of Warsaw, Poland

(D) https://orcid.org/0000-0003-2427-0489

\section{Katarzyna Pelewicz}

Department of Internal Medicine and Endocrinology, Medical University of Warsaw, Poland

(iD) https://orcid.org/0000-0002-2333-2337

\section{Piotr Miśkiewicz}

Department of Internal Medicine and Endocrinology, Medical University of Warsaw, Poland

(iD) https://orcid.org/0000-0003-4015-6491

Corresponding author:pmiskiewicz@wum.edu.pl
DOI: https://doi.org/10.20883/medical.e519

Keywords: Graves orbitopathy, dysthyroid optic neuropathy, quality of life, visual acuity, diplopia

Published: 2021-06-28

How to Cite: Pelewicz M, Rymuza J, Pelewicz K, Miśkiewicz $P$. Impact of additional intravenous methylprednisolone pulse therapy on the quality of life in patients with dysthyroid optic neuropathy. JMS. 2021 Jun. 28;90(2):e519. doi:10.20883/medical.e519

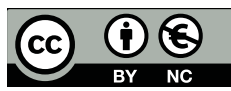

(C) 2021 by the author(s). This is an open access article distributed under the terms and conditions of the Creative Commons Attribution (CC BY-NC) licencse. Published by Poznan University of Medical Sciences

\begin{abstract}
Introduction. Dysthyroid optic neuropathy (DON) is a severe complication of Graves' orbitopathy (GO). Treatment of DON should involve immediate administration of intravenous methylprednisolone (ivMP) in very high doses. It is recommended to include additional 12 pulses of ivMP according to a weekly schedule as a further step of the treatment process. The purpose of this study was to evaluate the influence of a 12-week ivMP treatment on the quality of life (QoL) in DON patients.

Material and Methods. A retrospective study was conducted on 6 patients (the tests involved 8 individual eyes) with DON and treated with ivMP in very high doses, followed by orbital decompression in one patient. All patients were qualified for additional treatment with ivMP in a 12-week protocol and completed the Polish version of the GOQoL questionnaire before and after the therapy. Visual acuity (VA) and diplopia were examined prior to the administration of ivMP pulses for DON, as well as before and after the additional ivMP treatment. Results. A minimal clinically important difference in QoL was observed in four patients at the end of the additional ivMP therapy. A significant increase in VA was observed following additional pulses of ivMP compared to the evaluation at the time of the DON diagnosis $(p=0.04)$.

Conclusions. Applying additional 12 pulses of ivMP following DON therapy may impact QoL. Performing QoL assessment throughout the entire therapy in patients with DON is particularly important in the clinical practice. Final evaluation of QoL should be performed after completing the entire therapeutic process, which involves surgical treatment to correct diplopia.
\end{abstract}

\section{Introduction}

Graves' orbitopathy (GO) is an autoimmune orbital disorder manifested by disfiguring proptosis, diplopia, pain, redness and swelling of the eyelids $[1,2]$. The pathogenesis is based on inflammation, adipogenesis, and the production of glycosaminoglycans, which may lead to the expansion of the 
orbital connective tissue and the enlargement of the eye muscles [3]. Approximately $5 \%$ of the GO patients suffer from dysthyroid optic neuropathy (DON). This sight-threatening complication results from optic nerve compression caused by swollen muscles and fat in the orbital apex $[4,5]$. The first-line treatment of DON recommended by the European Group on Graves' Orbitopathy (EUGOGO) consists of intravenous methylprednisolone (ivMP) pulse therapy (500-1000 mg for 3 consecutive days). In the case of poor or absent response within 2 weeks, urgent orbital decompression should be performed. Furthermore, it is recommended that patients with a complete recovery receive additional treatment with 12 pulses of ivMP scheduled every week [6].

Up to now, there are no data to verify the influence of additional therapy with ivMP in a 12-week protocol on the quality of life ( $\mathrm{QoL}$ ) in DON patients. Nevertheless, it has been found that moderateto-severe GO negatively affects patients' wellbeing. Persons with GO suffer from poorer QoL, both with regard to vision problems and compromised appearance, compared to healthy individuals $[7,8]$. By means of the National Eye Institute Visual Function Questionnaire (NEI VFQ-25) Du Y et al. demonstrated that vision-related QoL tends to be more impaired in GO patients with DON than in those not suffering from DON [9]. However, NEI VFQ-25 is not a specific QoL questionnaire for patients with $\mathrm{GO}$, and it does not concern some of the unique issues affecting patients with $\mathrm{GO}$, such as altered appearance.

According to EUGOGO, the evaluation of QoL should constitute an integral part of management in patients with $\mathrm{GO}$. Therefore, it is recommended to use specific GOQoL questionnaire, which has been proven to be valid, reliable, and culturally applicable[10]. In 2015 a validated Polish version of the GOQoL questionnaire (GOQoLpl) was developed and subsequently published by EUGO$\mathrm{GO}$ as the recommended version for the assessment of QoL among Polish patients with GO in the clinical practice [11]. Since the development of the GOQoLpl only a handful of studies evaluating QoL of GO patients have been conducted, and no study has been performed regarding DON.

The purpose of this study was to evaluate the impact of additional ivMP treatment in a 12-week protocol on the quality of life of DON patients.

\section{Material and Methods}

\section{Patients}

Six individuals diagnosed with DON were retrospectively recruited in the study. A total number of 8 eyes were affected by DON. Patients were treated in the Department of Internal Medicine and Endocrinology, Medical University of Warsaw, between 2015 to 2018. The diagnosis of DON was based on at least two features, such as deterioration of visual acuity (VA) $(<1.0)$ and/or colour vision, optic disc swelling and/or signs of DON in magnetic resonance imaging (optic nerve stretching and/or presence of apical crowding) [12]. The inclusion criterion was an additional treatment with ivMP in a 12-week protocol following the treatment of DON. The exclusion criteria were: a previous history of ivMP therapy for $\mathrm{GO}$, as well as a lack of a completed GOQoL questionnaire before or after the additional treatment with ivMP.

\section{Treatment}

All patients were administered ivMP $(3 \times 1.0 \mathrm{~g}$ given within three consecutive days). Due to the poor improvement one individual received additional pulses of ivMP $(3 \times 1.0 \mathrm{~g}$ and $3 \times 0,5 \mathrm{~g}-$ cumulative dose of $8 \mathrm{~g}$ ) and another one underwent additional endoscopic intranasal orbital decompression. Subsequently each patient was qualified for treatment with 12 pulses of ivMP. Five patients received an additional cumulative dose of $4.5 \mathrm{~g} \mathrm{(} 0.5 \mathrm{~g}$ once weekly for 6 weeks, followed by $0.25 \mathrm{~g}$ once weekly for 6 weeks). One patient (treated with $8 \mathrm{~g}$ IVMP for DON) was qualified for 12 pulses of ivMP with a cumulative dose of $7.5 \mathrm{~g}$ ( $0.75 \mathrm{~g}$ once weekly for 6 weeks, followed by $0.5 \mathrm{~g}$ once weekly for 6 weeks), but due to the increased level of alanine and aspartate aminotransferases this patient received 9 pulses of ivMP.

\section{Laboratory and ophthalmic evaluation}

The serum levels of thyroid-stimulating hormone (TSH), free triiodothyronine (fT3), free thyroxine (fT4) and TSH binding inhibitory immunoglobulin (TBII) were evaluated with an electro-chemiluminescent immunoassay performed on a Cobas 6000 analyser from Roche Diagnostics (Mannheim, Germany).

VA of the patients' eyes diagnosed with DON was verified using Snellen charts and expressed 
as a decimal fraction. The Gorman score was used to evaluate and classify diplopia graded from 0 - 3: 0-no diplopia, 1-intermittent diplopia, 2-inconstant diplopia, 3-constant diplopia [13]. Each parameter was assessed at three time points: prior to the administration of the first-line treatment with ivMP pulses for DON, before and after the additional ivMP treatment.

\section{QoL questionnaire}

At the beginning of and after the treatment with additional pulses of ivMP, all patients completed GOQoLpl. It consists of two subscales which assess: limitations in visual functioning ( 7 questions) and influence of the GO on appearance (8 questions). Each question is scored based on a 3-point scale referring to GO impact: 1-serious, 2-little, 3-no impact. The results are established on the basis of the following formula: (total score- \#)/(2 × \#) $\times 100$ where \# indicates the number of completed items. The total QoL score is expressed as a number between 0 to 100 , where a higher result indicates better QoL.

According to Wiersinga et al., a minimal clinically important difference (MCID) in the GOQoL for immunosuppression and surgical decompression is a change of $\geq 10$ points [14].

\section{Statistical analysis}

All the analyses were performed using SPSS statistical software version 22.0 (IBM SPPS Statistics, New York, US). The results were expressed as a mean ( \pm standard deviation) except for the GOQoL score, which was expressed as a median (interquartile range). Categorical variables were expressed as numbers (n) and percentages (\%). The Shapiro-Wilk test was applied to confirm or reject the normal distribution of each continuous variable. Comparisons between continuous data were performed using a paired t-test (for parameters with normal distribution), or the Wilcoxon rank sum test (for parameters with the distribution deviations). Statistical significance was established for the results with $p<0.05$.

\section{Results}

The demographic details and clinical characteristics are presented in Table 1. DON was diagnosed in 6 patients in the total of 8 eyes ( 2 patients with bilateral DON and 4 patients with unilateral DON). VA significantly increased following the additional treatment with ivMP compared to the baseline evaluation at the time of the diagnosis of DON

Table 1. Baseline demographic and clinical characteristics at three time points: prior to the administration of ivMP for DON, before and after the additional treatment with ivMP pulses

\begin{tabular}{|c|c|c|c|}
\hline \multicolumn{4}{|l|}{ Demographics } \\
\hline Age, years ${ }^{a}$ & \multicolumn{3}{|c|}{$69.33( \pm 5.79)$} \\
\hline Male/female ${ }^{b}$ & \multicolumn{3}{|c|}{$2 / 4$} \\
\hline \multirow[t]{2}{*}{ Number of eyes with DON } & \multicolumn{3}{|c|}{8} \\
\hline & 0 & I PULSE & LAST PULSE \\
\hline \multicolumn{4}{|c|}{ Clinical characteristics of thyroid disease } \\
\hline TSH $(0.27-4.2 \mu \mathrm{lU} / \mathrm{mL})^{\mathrm{a}}$ & $1.14( \pm 1.42)$ & $0.68( \pm 0.52)$ & $1.21( \pm 0.79)$ \\
\hline fT3 $(3.1-6.8 \mathrm{pmol} / \mathrm{L})^{\mathrm{a}}$ & $4.64( \pm 1.80)$ & $4.66( \pm 1.33)$ & $4.33( \pm 0.94)$ \\
\hline fT4 (12-22 pmol/L) ${ }^{\mathrm{a}}$ & $19.06( \pm 4.28)$ & $15.72( \pm 2.61)$ & $17.37( \pm 3.33)$ \\
\hline TBII $(<1.75 \text { IU/L) })^{\mathrm{a}}$ & $10.44( \pm 7.17)$ & $6.24( \pm 4.12)$ & $3.47( \pm 2.53)$ \\
\hline \multicolumn{4}{|c|}{ Clinical characteristics of orbital disease } \\
\hline \multicolumn{4}{|c|}{ Gorman score $^{\mathrm{b}}$} \\
\hline No diplopia & 3 & 1 & 2 \\
\hline Intermittent diplopia & 1 & 1 & 1 \\
\hline Inconstant diplopia & 1 & 1 & 1 \\
\hline Constant diplopia & 1 & 3 & 2 \\
\hline Visual acuity ${ }^{\mathrm{a}}$ & $0.58( \pm 0.27)$ & $0.78( \pm 0.29)$ & $0.83( \pm 0.23)$ \\
\hline
\end{tabular}


$(p=0.04)$. A significant improvement has not been observed either between the assessment prior to the treatment of DON and the beginning of the additional therapy $(\mathrm{p}=0.125)$, or between the $1^{\text {st }}$ and the last pulse of the additional therapy $(p=0.18)$. Mean values of VA are shown in Figure 1.
All six patients completed GOQoLpl. The median GOQoLpl total score, as well as subtotals for visual functioning and appearance before and after the ivMP treatment are provided in Figure 2. MCID was observed in four patients (2 patients - an improvement of QoL, 2 patients - a dete-

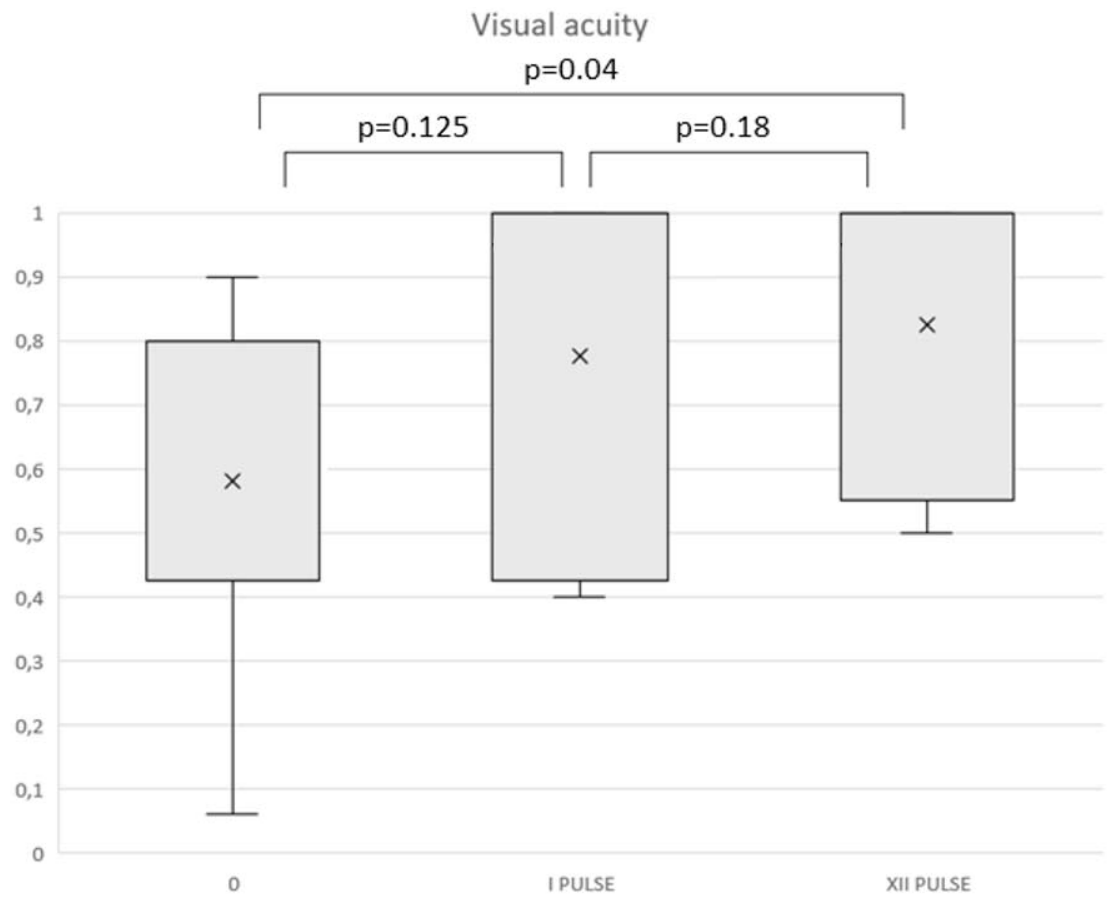

Figure 1. Visual acuity of 8 eyes in patients diagnosed with dysthyroid optic neuropathy (DON). A comparison of variables between the time of the diagnosis of DON $(0)$, the $1^{\text {ST }}$ and the last pulse of additional intravenous methylprednisolone treatment. Vertical line ranges from a maximum to a minimum value. Bold square presents a standard deviation. $\mathrm{x}-$ mean

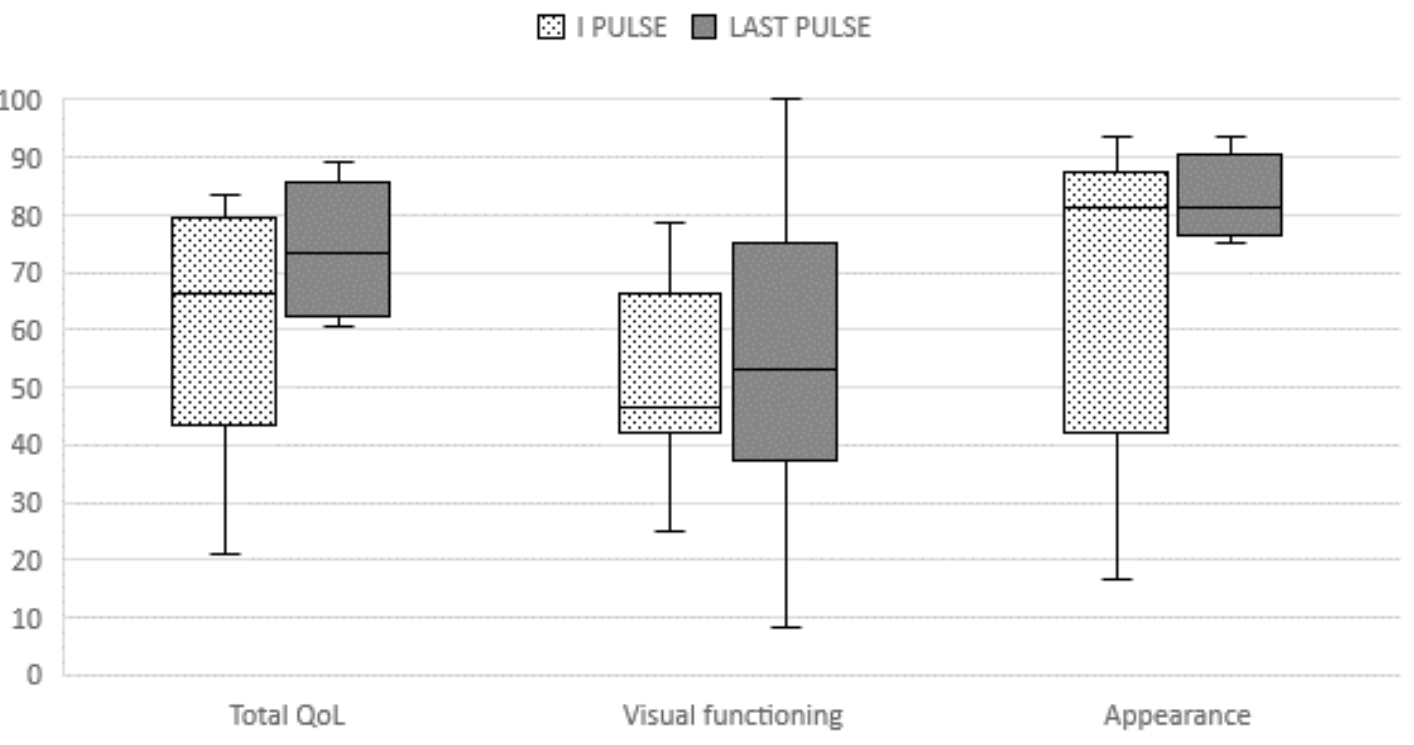

Figure 2. Total Graves' Orbitopathy Quality of Life $(\mathrm{Q} O \mathrm{~L})$ questionnaire score and its subscales: visual functioning and appearance were based on the following formula: (total score- \#)/(2×\#) $\times 100$ where \# indicates the number of completed items. The total QoL score varies from 0 to 100. Higher result indicates better QoL. A comparison of variables between the $1^{\mathrm{ST}}$ and the last pulse of intravenous methylprednisolone treatment. Vertical line ranges from maximum to minimum value. Data are shown as median values (line across the box) with interquartile $\left(25^{\text {th }}-75^{\text {th }}\right.$ percentile) range (the box) 
rioration of $\mathrm{QoL})$. In two patients MCID was not detected.

One of the patients with a deterioration of QoL after the additional treatment, who initially exhibited monocular vision in the course of DON, developed diplopia, due to the improvement of VA during the additional treatment. Furthermore, we found a reduction of eye motility with a deterioration of diplopia in another patient.

\section{Discussion}

According to the EUGOGO, the treatment of DON involves immediate administration of ivMP in very high doses followed by urgent orbital decompression as a second-line treatment in the case of poor, or absent response within 2 weeks. Subsequently, patients with a complete recovery should be qualified for additional ivMP pulses in a 12-week protocol [6]. The main purpose of the additional therapy is to maintain the clinical improvement and minimize the inflammatory process. Nevertheless, its impact on QoL of the patients with DON has not been investigated until now.

Our study, performed on 6 patients with DON, showed no significant influence of the additional ivMP therapy on QoL. Simultaneously, an improvement in VA following the treatment was observed. A detailed analysis revealed that a decreased QoL after the additional treatment was associated with an exacerbation of diplopia and reduced motility of the eye muscles, which possibly deteriorated QoL, despite the improved VA. Furthermore, in some cases, the use of ivMP in high doses may lead to various side effects which can also interfere with QoL following the treatment [15], although generally it is considered to be highly efficient and mostly safe [16].

\section{Results}

The results of this study indicate that an additional 12-week ivMP treatment should constitute an integral part of the strategy in the management of DON. In terms of analysing a comprehensive therapy of DON, we should also consider involving rehabilitative surgery of the extraocular muscles. As demonstrated in our study, some patients consider the presence of diplopia to be a more relevant factor when assessing QoL, rather than improved VA. Therefore, strabismus surgery may have a positive impact on QoL.

To our knowledge, it is the first report evaluating changes in QoL in DON patient following the treatment with 12 pulses of ivMP. The main limitations of our study are its retrospective character and the small sample size. Nevertheless, the following conclusions may be drawn.

\section{Conclusions}

Including the additional 12 pulses of ivMP into combined therapy of DON may impact QoL. The assessment of QoL is, therefore, particularly important and should constitute an integral part of routine clinical practice. For some patients, diplopia or decreased eye motility become the main factor deteriorating QoL following the therapy, despite increased VA. The final evaluation of QoL should be performed after completing the entire therapeutic process, which involves surgical treatment to correct diplopia.

Following the first and second-line treatments of DON, a multidisciplinary and individual approach is necessary to maximize the potential improvement of signs and symptoms of the disease.

\section{Acknowledgements}

\section{Conflict of interest statement}

The authors declare no conflict of interest.

\section{Funding sources}

There are no sources of funding to declare.

\section{References}

1. Burch HB, Wartofsky L. Graves' ophthalmopathy: Current concepts regarding pathogenesis and management. Endocrine Reviews. 1993;14(6):747-793. https:// doi.org/10.1210/edrv-14-6-747 PMID 10.1210/edrv-14 $-6-747$

2. Weetman AP. Thyroid-associated eye disease: pathophysiology.. Lancet (London, England). 1991 Jul;338(8758):25-28. https://doi.org/10.1016/01406736(91)90013-f PMID 10.1016/0140-6736(91)90013-f

3. Wiersinga WM. Autoimmunity in Graves' ophthalmopathy: The result of an unfortunate marriage between TSH receptors and IGF-1 receptors?. Journal of Clinical Endocrinology and Metabolism. 2011;96(8):23862394. https://doi.org/10.1210/jc.2011-0307 PMID 10.1210/jc.2011-0307

4. Neigel JM, Rootman J, Belkin RI, Nugent RA, Drance SM, Beattie CW, Spinelli JA. Dysthyroid optic neu- 
ropathy. The crowded orbital apex syndrome.. Ophthalmology. 1988 Nov;95(11):1515-1521. https://doi. org/10.1016/s0161-6420(88)32978-7 PMID 10.1016/ s0161-6420(88)32978-7

5. Kennerdell JS, Rosenbaum AE, El-Hoshy MH. Apical opticnervecompression of dysthyroid opticneuropathy on computed tomography.. Archives of ophthalmology (Chicago, Ill.: 1960). 1981 May;99(5):807-809. https:// doi.org/10.1001/archopht.1981.03930010807002 PMID 10.1001/archopht.1981.03930010807002

6. Bartalena L, Baldeschi L, Boboridis K, Eckstein A, Kahaly GJ, Marcocci C, Perros P, Salvi M, Wiersinga WM. The 2016 European Thyroid Association/European Group on Graves' Orbitopathy Guidelines for the Management of Graves' Orbitopathy.. European thyroid journal. 2016 Mar;5(1):9-26. https://doi. org/10.1159/000443828 PMID 10.1159/000443828

7. Gerding MN, Terwee CB, Dekker FW, Koornneef L, Prummel MF, Wiersinga WM. Quality of life in patients with Graves' ophthalmopathy is markedly decreased: measurement by the medical outcomes study instrument.. Thyroid: official journal of the American Thyroid Association. $1997 \mathrm{Dec} ; 7(6): 885-889$. https://doi.org/10.1089/ thy.1997.7.885 PMID 10.1089/thy.1997.7.885

8. Kulig G, Andrysiak-Mamos E, Sowińska-Przepiera E, Kulig J, Karakiewicz B, Brodowski J, Robaczyk M, Homa K, Letkiewicz M, Syrenicz A. Quality of life assessment in patients with Graves' disease and progressive infiltrative ophthalmopathy during combined treatment with methylprednisolone and orbital radiotherapy.. Endokrynologia Polska. 2009;60(3):158-165.

9. Du Y, Ye H, Li K, Xiao X, Chen R, He J, Yang H. Visionrelated quality of life tends to be more severely impaired in patients with dysthyroid optic neuropathy.. Current eye research. 2014 May;39(5):532-536. https://doi.org/10.3109/02713683.2013.848901 PMID 10.3109/02713683.2013.848901

10. Lee THB, Sundar G. Quality of Life in Thyroid Eye Disease: A Systematic Review.. Ophthalmic plastic and reconstructive surgery. 2020;36(2):118-126. https://
doi.org/10.1097//OP.0000000000001446

PMID 10.1097/IOP.0000000000001446

11. Sawicka-Gutaj N, Bednarczuk T, Daroszewski J, Waligórska-Stachura J, Miśkiewicz P, Sowiński J, Bolanowski M, Ruchała M. GO-QOL--disease-specific quality of life questionnaire in Graves' orbitopathy.. Endokrynologia Polska. 2015;66(4):362-366. https://doi.org/10.5603/EP.2015.0046 PMID 10.5603/ EP.2015.0046

12. Miśkiewicz $P$, Rutkowska $B$, Jabłońska A, Krzeski $A$, Trautsolt-Jeziorska K, Kęcik D, Milczarek-Banach J, Pirko-Kotela K, Samsel A, Bednarczuk T. Complete recovery of visual acuity as the main goal of treatment in patients with dysthyroid optic neuropathy. Endokrynologia Polska. 2016;67(2):166-173. https:// doi.org/10.5603/EP.a2016.0018 PMID 10.5603/ EP.a2016.0018

13. Bahn RS, Gorman CA. Choice of therapy and criteria for assessing treatment outcome in thyroid-associated ophthalmopathy.. Endocrinology and metabolism clinics of North America. 1987 Jun;16(2):391-407.

14. Wiersinga WM, Prummel MF, Terwee CB. Effects of Graves' ophthalmopathy on quality of life.. Journal of endocrinological investigation. $2004 \mathrm{Mar}$;27(3):259264. https://doi.org/10.1007/BF03345275 PMID 10.1007/BF03345275

15. Vogel A, Elberling T, Hørding M, Dock J, Rasmussen AK, Feldt-Rasmussen U, Perrild H, Waldemar G. Affective symptoms and cognitive functions in the acute phase of Graves' thyrotoxicosis.. Psychoneuroendocrinology. 2007 Jan;32(1):36-43. https://doi. org/10.1016/j.psyneuen.2006.09.012 PMID 10.1016/j. psyneuen.2006.09.012

16. Miśkiewicz $P$, Kryczka A, Ambroziak U, Rutkowska B, Główczyńska R, Opolski G, Kahaly G, Bednarczuk $T$. Is high dose intravenous methylprednisolone pulse therapy in patients with Graves' orbitopathy safe?. Endokrynologia Polska. 2014;65(5):402-413. https://doi.org/10.5603/EP.2014.0056 PMID 10.5603/ EP.2014.0056 